\title{
Early defect diagnosis in installed PV modules exploiting spatio-temporal information from thermal images
}

\author{
by S.Rogotis*, D.loannidis***, A. Tsolakis*, D.Tzovaras*, S.Likothanassis ${ }^{\star \star}$
}

*Information Technologies Institute - Centre for Research and Technology Hellas, ThermiThessaloniki, 57001, Greece, \{srogotis, djoannid, tsolakis, dimitrios.tzovaras\}@iti.gr

**Pattern Recognition Laboratory - Computer Engineering and Informatics, University of Patras, Rio, Patras, Greece, \{djoannid, likothan\}@ceid.upatras.gr

\begin{abstract}
Photovoltaic (PV) technology has evolved rapidly during the last decade and alongside the interest for the identification of the key factors that affect PV modules' performance during operation. Among different preventive maintenance techniques, thermal imaging offers considerable advantages regarding equipment's condition monitoring as it provides a nondestructive and cost-effective inspection tool. This paper attempts to address the problem of early defect diagnosis at installed PV modules through the exploitation of spatio-temporal information from thermal images. The proposed method aims to minimize standard maintenance costs and reduce the necessity of human intervention.
\end{abstract}

\section{Introduction}

During the last decades, renewable energy sources (RES) have entered the spotlight since they present great potential in solving the world's energy problem and in reducing the dependence to fossil fuels. The latter, raise serious environmental concerns due to the resulting high carbon dioxide emissions that lead to global warming. Among different technologies, solar energy offers major advantages on the basis of sustainability, safety and environmental benefits. Consequently, PV systems progressively became irreplaceable contributors in world's energy mix through the exploitation of sunlight which is the largest available energy source known to mankind. However, in order to ensure their sustainability, it is deemed essential to optimize their performance by reducing any faults, defects or malfunctions prior or after the installation stage. Condition monitoring techniques focusing on the manufacturing phase of the PV modules exhibit adequately maturity, yet there are still defects that may pass undetected. Alongside, operating PV modules are subjected to long-term outdoor exposure in diversified environmental conditions. This often causes additional degradation due to several factors such as exposure to mechanical stresses and UV radiation, air pollution, increased humidity, high ambient temperatures, module aging, etc. As such, preventive maintenance (PM) or predictive maintenance (PdM) techniques should be carried out in regular bases on the field in order to increase the equipment availability and secure the installation from defects of greater gravity such as hot-spots. Undetected defects or malfunctions might downgrade the system overall performance and therefore the investment profitability.

This paper proposes an early defect diagnosis method designed for in-situ monitoring of PV modules by means of thermal imaging. It is divided into six main sections. The initial section has a preliminary nature as it states the problem that our method aspires to resolve. Section 2 consists of a detailed literature review of methods and techniques that allow the detection of defected PV areas including industrially standardized approaches and vision-based techniques. The proposed methodology is being described in Section 3. The latter is further divided into two sub-sections that cope with PV module localization and defect diagnosis using Panel Energy Image (PEI) method respectively. Section 4 presents the experimental set-up while section 5 discusses the experimental results along with comparisons with baseline methods. Finally, in section 6 the paper conclusions are drawn.

\section{Previous Work}

Standard condition monitoring techniques in PV plants involve I-V curve analysis and performance measurements of whole PV strings or arrays [1]. This is due to the immense expense and time expenditure needed for a detailed electrical analysis of each PV module. Moreover, these methods often meet several limitations on occasions where the measurement conditions are not optimal. For example, the Maximum Power Point Tracking (MPPT) method cannot track the real MPP under partially shaded conditions [2]. To this end, various non-destructive techniques have been developed over the past few years, among which thermal imaging is considered as a very promising, fast, costeffective and highly reliable method for the detection of defected or underachieving areas [3].

According to Planck's law for black body radiation, all objects emit infrared radiation which is proportional to their temperature. Thermal cameras translate the incident radiation into static monochromatic or color images, determining thus, the thermal distribution across the inspected surface and allowing the detection of hidden abnormalities that are not easily detectable with conventional inspection methods. Based on effective black body radiance at given temperature ranges the IR systems can be thermographically calibrated to measure surface temperatures. Thermal imaging and infrared thermography (IRT) are divided into two large categories: active and passive [4].

The active approach supposes that the inspected PV modules have physical interaction with the measuring setup (e.g. stimulations pulses) that augments the temperature differences between the sound and the defected areas. As a result, accurate information regarding any abnormalities in the surface, structure, connections and soldering can be easily extracted. Due to its intrusive nature, the active approach is not suitable for in situ monitoring of PV modules and mainly concentrates on the detection of defects originating from the manufacturing process. Thereby, it is most commonly applied prior to any field installation. Lock-in thermography is a very promising active approach that requires 
periodic heating on a specific frequency in the interior of the inspected module $[5,6]$. The resulting phase delay and peak distortion are measured from distance to evaluate the module's health. Besides active IRT, industrial stakeholders often utilize alternative methods for equipment inspection prior to installation. Electroluminescence and photoluminescence methods employ internal electrical stimulation to the PV modules and perform health assessment procedures by exploiting wavelengths close to the infrared spectrum $[7,8]$. Hyperspectral imaging is another inspection method that relies on the fact that PV modules are manufactured by materials and surfaces that reflect at least $60 \%$ of any incident wave. The presence of exceptionally low reflection percentage might indicate undying defects [9].

Passive approach, on the other hand, does not involve interaction between the measuring setup and the PV modules since the inspected equipment is already adequately stimulated. The operating temperature of a healthy PV cell is directly linked to certain environmental conditions and more specifically to wind speed, solar irradiance and ambient temperature [10]. When a PV cell is malfunctioning, its efficiency in energy production is considerably degraded. Since a single PV module encompasses many series-connected cells, the defective cell is forced to operate outside its capabilities and gradually becomes reversed-biased. Thereby, it operates as an energy consumer by dissipating large amounts of produced energy to the environment in the form of heat. The possibly defected areas share a significantly different temperature profile than the sound areas enabling their detection by means of thermal imaging. The use of hand-held thermographic cameras gained popularity during the past few years providing fast and reliable inspections (figure 1). However, the presence of an experienced thermographer on the field still remains a prerequisite [11,12], considering that quite a few factors need to be taken into account for a valid outcome[13] (reflections, weather conditions, etc.). In order to minimize the influence of those factors on the acquired images, the PV modules can be inspected from the rear side as well. These non-automatic techniques offer quantitative results using spatial information. Line profile analysis, tridimensional temperature diagrams and intensity histograms are used to highlight the defected areas. Advances in IR technology have led to IR cameras that can take as input several parameters such as emissivity, measurement distance and certain environmental factors, like ambient temperature, humidity, wind speed and reflective temperature, making the task of the thermographer a lot easier.

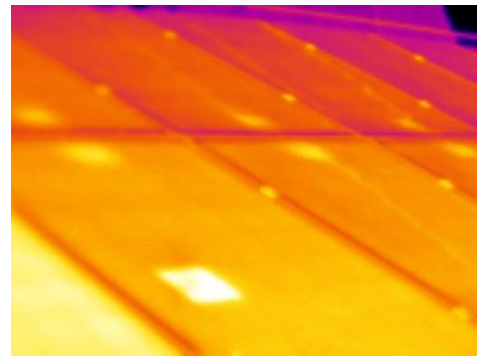

a) Inspection from the front side

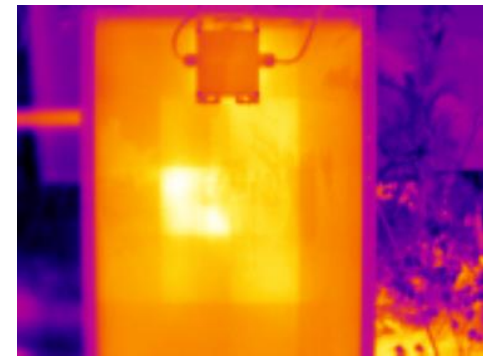

b) Inspection from the rear side

Fig. 1. Manual IRT inspection of PV modules. Abnormal color patterns indicate possible defects

Over the years, only few research efforts have concentrated on the development of robust image processing algorithms that are able to extract meaningful information from thermal images. These algorithms are mostly based on image segmentations by means of thresholding techniques borrowed from color images. Thresholding is often applied for background separation and object detection. The original image is being separated in two classes according to a global or local threshold value. An important tool for constructing the binary image is the gray-level histogram of the original image. Many thresholding techniques have been introduced in the literature that differentiate themselves by determining the threshold value based on different histogram or alternative criteria [14]. Among others, otsu thresholding approach is a very popular global thresholding technique that computes the optimal threshold value so as to maximize the separability of the resultant classes in gray levels [15]. The Otsu method is widely accepted because of its simplicity and its capability to effectively separate bimodal images. These techniques, although not originally addressed for thermal images have provided the foundation towards the development of an efficient segmentation method that can be applied for the separation of region of interests (ROIs) in infrared imagery.

The ROIs in thermal images usually share distinctive patterns and can be visually discriminated from the rest of the background since they appear as relatively bright or dark regions. The first research efforts tried to segment the thermal image and to extract blobs with the desired information [16], or used first-order statistic properties for warm object extraction and characterization [17]. These methods showed interesting results but where accompanied by limitations on their methodology, rendering them semi-automatic. Few years later, a different approach introduced a twostep algorithm that primarily locates all defects (seeds) and then applies a region-growing method using a proper thresholding value [18]. Once again, the method is not fully automatic since some of the necessary values are given as input by the user. Those limitations were partially removed with the development of an automatic thresholding selection algorithm [19]. Moreover, notable results were also provided through the utilization of the wavelet method for a multi-level thresholding algorithm $[20,21]$. Other research efforts focused on textural features [22], 2-D and multi-dimensional histograms [23,24] and entropy based algorithms [25,26]. In 2009 two different research teams have developed rather promising algorithms, emphasizing on automatic abnormality detection of defective equipment in thermal images. The first one was the Infrared Thermography Anomaly Detection Algorithm (ITADA) [27], that employs segmentation and region-growing techniques in thermal images presenting defective equipment and results in a $91 \%$ accuracy thus, providing a valuable tool in preventive maintenance. The second approach proposed a multi-level contrast enhancement 
algorithm that can distinguish either "hot" defects (Intensity Brightening Operation - IBO) or "cold" defects (Intensity Darkening Operation - IDO) over petrochemical equipment. For the discrimination process it follows simple histogrambased thresholding techniques [28]. However, both the aforementioned algorithms are designed for single-image operations and as such they fail in cases where reflections severely corrupt the image quality.

Within this paper, we propose a condition monitoring method for installed PV modules that is based on passive IR approach. Our method operates differently than existing techniques as it combines industrially standardized inspection tools with image processing approaches in order to diagnose potential system threats in an automatic perspective. Except from the effective localization of the PV modules that lay inside the camera's field of view, we attempt to address the problem of defect detection in PV modules that are often subjected to specular reflections and noise. Our major contribution in this context is that we use both spatial and temporal information in order to monitor the defections evolvement over time on the basis that reflections and other incorrectly tracked areas tend to fade after a period of time. Additionally, we integrate environmental and neighborhood-based aspects towards assessing the ability of each PV module to be evaluated for possible defects. This policy ensures that our method exhibits resistance to spatial and temporal noise that is often encountered during IR inspections.

\section{Methodology}

\subsection{PV Module Localization}

The first stage of the proposed methodology is aiming at the localization of the PV modules that lay inside the IR camera's field of view. Our methodology begins with a segmenting operation using Otsu threshold [15]. Otsu thresholding is particularly effective when dealing with images that are bimodal in nature. To this end, the localization process is being performed during nighttime when the captured images present a rather balanced foregroundbackground distribution. Generally speaking, the radiation that originates from the PV modules consists of three individual components (figure 2).

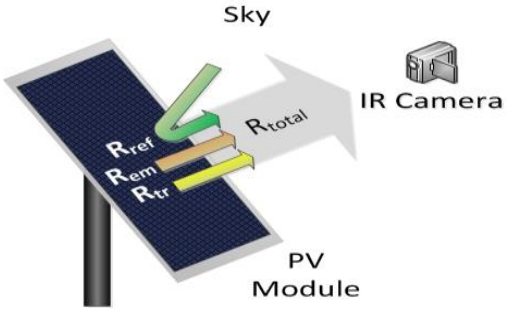

Fig. 2. The individual radiation components that incident on the IR camera's detector

During nighttime, the outgoing radiation from the PV modules consists of the radiation that is being emitted by their cold surfaces $\left(R_{e m}\right)$, the radiation that is being transmitted through them $\left(R_{t r}\right)$, and the reflection of the "cold" sky $\left(R_{\text {ref }}\right)$. The transmitted radiation can be omitted since it doesn't play a significant role in practice. As a result, the inspected PV modules appear as cold/dark objects that can be easily separated from the surrounding environment. Since the metallic frames that surround the PV modules have different properties than the protective surface glasses, the outer borders of the inspected PV modules can also be distinguished (figure 3a). Otsu thresholding leads to a binary image that includes all candidate regions that correspond to PV modules (figure $3 \mathrm{~b}$ ). In order to separate these regions and discard false detections, morphological operations are further applied [29] (figure 3c).

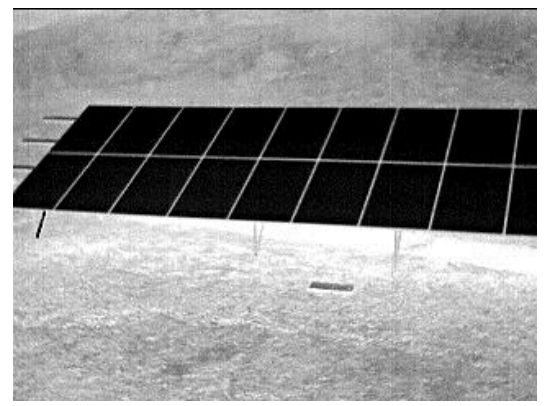

a) Original image

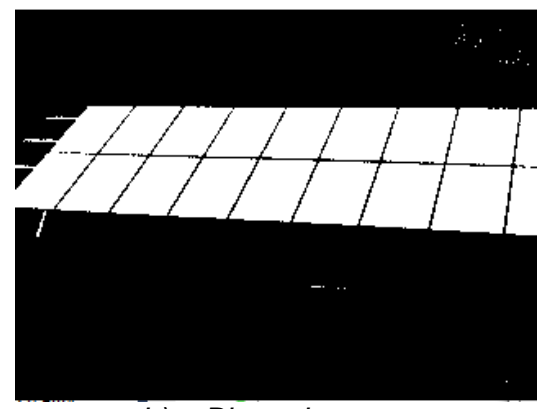

b) Binary image

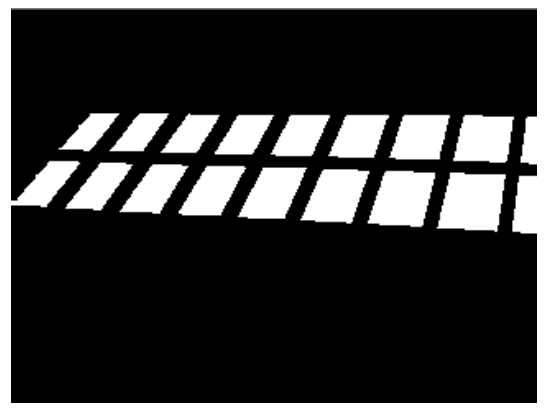

c) Segmented image

Fig. 3. Segmentation overview

The next step of the localization process is targeting at refining the PV modules' boundaries. To accomplish that a line profile analysis is applied between the centers of mass of all neighboring modules. This is based on the idea that the two largest intensity fluctuations across the line that connects the centroids of two neighboring PV modules is highly possible to indicate the position of their boundaries (figure 4). The centers of mass are calculated from the segmented image of the previous step whereas the line profile analysis is performed at the original image. For the special cases that neighboring modules are not existing, the proposed algorithm searches for the highest intensity fluctuation in a search window equal to the module's diagonal that is calculated from its rotated bounded box. At the end of the process, the returned boundary points, produce a set of grid lines using the list squares line fitting algorithm [30]. The intersections 
between the grid lines define the position of the PV modules' vertexes and consequently their boundaries (figure 5). It is evident that PV modules that only partially appear and don't provide information that is required to build the grid lines are discarded (figure 5c).

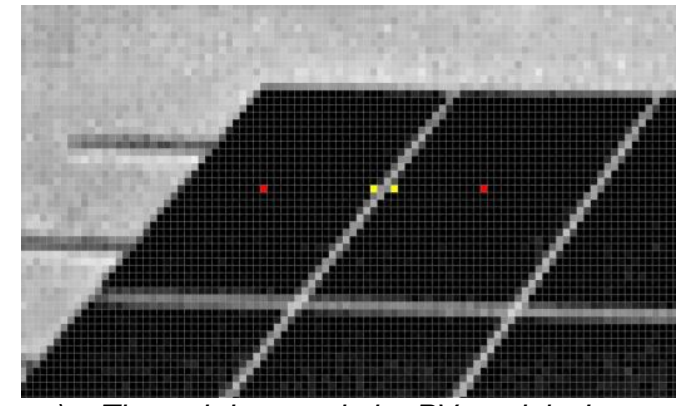

a) The red dots mark the PV modules' centroids while the yellow dots their boundary points

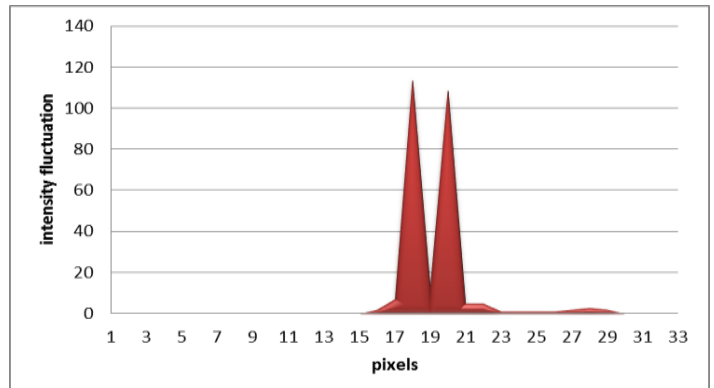

b) Intensity fluctuations across the line that connects the PV modules' centroids

Fig. 4. Line profile analysis between neighboring PV modules

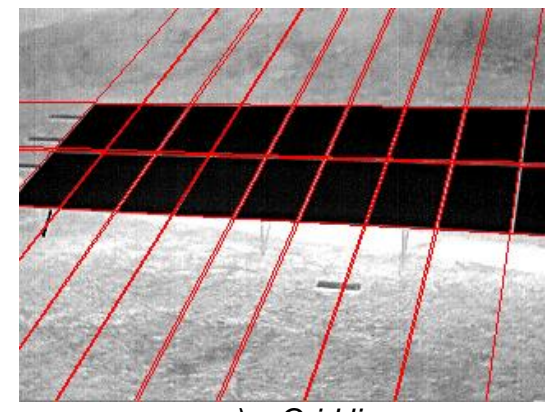

a) Grid lines

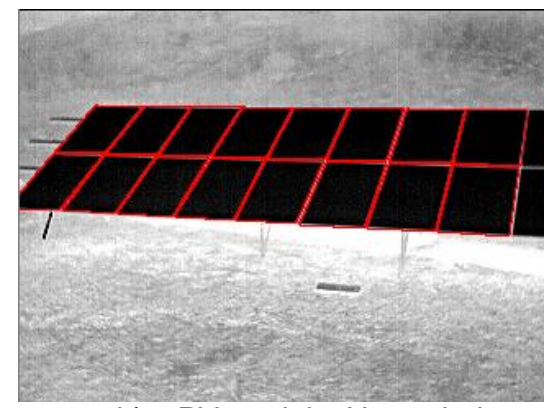

b) PV modules' boundaries

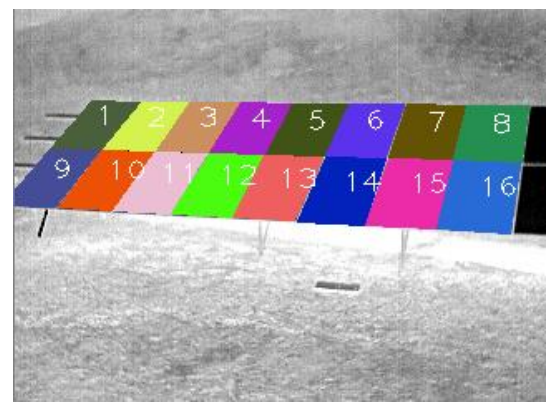

c) PV modules'labels

Fig. 5. Localization and labelling process

\subsection{Panel Energy Image Method}

While the localization process is performed during nighttime, defect detection is conducted in the time interval from sunrise to sunset, when the electricity generation procedure is at its peak and the modules are adequately stimulated allowing the detection of defected or malfunctioning areas.

For every input frame, rectangular thermal maps are being extracted that correspond to the inspected PV modules. Their boundaries are being used to create binary masks that allow only relevant information to take part in the condition monitoring procedure. As long as certain conditions are fulfilled, the evaluation can be further continued. These conditions are divided into two groups: individual and holistic conditions. Holistic conditions should be constantly fulfilled in order to ensure that the PV modules are adequately stimulated and the environmental impact is within acceptable ranges for effective IR inspections. On the other hand, individual conditions refer to specific parameters which indicate the quality of information that is being used for condition monitoring of each PV module. Experiments have shown that solar irradiance and wind speed are suitable holistic control variables, while the PV module's average intensity can be used as a simple but rather efficient individual control parameter.

Table 1 Holistic and Individual conditions that need to be fulfilled based on empirical results

\begin{tabular}{|c|c|c|c|}
\hline \multicolumn{2}{|c|}{ Holistic Conditions } & \multicolumn{2}{|r|}{ Individual Conditions } \\
\hline Solar Irradiance $\left(\mathrm{C}_{1}\right)$ & $>400 \mathrm{~m} / \mathrm{W}^{2}$ & Average & Between 25.6 (low 10\% threshold) \& \\
\hline Wind Speed $\left(\mathrm{C}_{2}\right)$ & $<6 \mathrm{~m} / \mathrm{s}$ & Intensity $\left(\mathrm{C}_{3}\right)$ & $\begin{array}{l}229.5 \text { (high } 10 \% \text { threshold) for an } \\
\text { image ranged from } 0 \text { to } 255 \text {. }\end{array}$ \\
\hline
\end{tabular}

The next phase of our approach targets at separating the sound (dark) from the defected (bright) areas by employing histogram stretching techniques. Primarily, the original thermal map (figure 6a) is subjected to a linear min-max normalization that enhances the image contrast. Then, a new image is produced based on the following operation:

$$
g_{(x, y)}=\left(\sum_{k=0}^{n} z_{k}\right)^{3}
$$

where $g(x, y)$ is the pixel at the new image and $z_{1}, z_{2}, \ldots, z_{n}$ are the n-neighbord pixels of pixel $z_{0}$ located at $(x, y)$ at the original image. This operation is based on the intensity brightening operation (IBO) originally introduced in [28]. In IBO's 
definition, however, $g(x, y)$ equals to the product of the pixels within a $3 \times 3$ mask. The underlying reason for this alteration is that multiplication is a more radical operation than addition and tends to drastically change the image histogram, transferring a considerable amount of intensity values at histogram maximum levels. This enables specular reflections that affect large PV module areas, to be misclassified as potential defections. Besides, since the methodology requires a bimodal histogram distribution, raising to the third power provides good empirical results. Alongside, the 8-neighborhood mask is maintained. A morphological dilation [29] ensures that the size of the defected areas is not diminished by the described procedure. The operation is being repeated for a second time in order to further enhance the image contrast (figure 6b, 6c). The image clearly exhibits a bimodal intensity distribution thus, allowing the segmentation of sound and defected areas by means of histrogram-based thresholding. Our method utilizes a global threshold that is computed by a combination of two threshold definition techniques initially introduced at [28]: Mean Relative Thresholding (MRT) and Mean Frequency Thresholding (MFT).

MRT is a simple threshold definition technique that uses the gradient of the image histogram which is defined as follows:

$$
g(i)=h(i+1)-h(i)
$$

where $g(i)$ is the gradient of the image histrogram $h(i)$ at the intensity level $i$. In order to compute the threshold, two values are required: $i_{H}$ and $i_{L .}$. These values can be calculated as such:

$$
\begin{gathered}
i_{L}=\left\{\begin{array}{ll}
g(i) & \text { if } g(i)>g(i-1) \text { and } g(i)>g(i+1) \text { and } g(i) \geq 0 \\
0 & \text { otherwise }
\end{array}\right\} \\
i_{H}=\left\{\begin{array}{ll}
g(i) & \text { if } g(i)<g(i-1) \text { and } g(i)<g(i+1) \\
\max & \text { otherwise }
\end{array}\right\}
\end{gathered}
$$

where max corresponds to the highest intensity level of the image histogram. The searching process for these values is performed within the ranges $[0, \max ]$ and $[\max , 0]$ respectively. As soon as the criteria from the equations (3) and (4) are fulfilled the search stops. Finally MRT is determined by:

$$
T h_{M R T}=\left\|\frac{i_{H}-i_{L}}{2}\right\|
$$

MFT on the other hand, searches the image histogram for the minimum intensity frequency. If this minimum intensity frequency is met more than once, the left-most level is defined as the appropriate threshold value.

$$
T h_{M F T}=\left.i\right|_{\min \left(h_{i}\right)} \mathrm{i}=0, \ldots ., \max
$$

In order to ensure that erroneous measurements don't place the threshold value very close to the lowest histogram extreme level and incorrectly classify large PV module areas as defected, the threshold values is bounded in the [Th $\mathrm{MRT}_{\text {, }}$ max] interval. Finally, the image is segmented by the following global threshold:

$$
T h=\left\{\begin{array}{ll}
T h_{M R T} & \text { if } T h_{M F T}<T h_{M R T} \\
T h_{M F T} & \text { otherwise }
\end{array}\right\}
$$

A morphological dilation [29] groups the pixels into clusters that signify candidate defected areas (figure 6d). In order to remove false detections three criteria must be fulfilled regarding area's size, shape and local intensity distribution. More specifically, small-sized and "ragged" contours are discarded. The latter indicates possible specular reflections that were incorrectly classified as potential defects. "Ragged" contours are eliminated according to a shape descriptor [31]:

$$
D=\frac{S}{l^{2}}
$$

where $S$ is the contour area, and $/$ is the contour length. In case that the value of the above descriptor falls beneath a pre-defined threshold the contour is discarded as a reflection. The third criteria refers to the local intensity distribution around the contour. Experiments have shown that when a contour represents a defected area, the standard deviation of the intensity across the contour is higher than the corresponding value inside or outside the contour. This is based on the idea that all defects exhibit a thermal gradient towards their boundaries as the developed temperature progressively 
decreases. To this end, each PV module is divided into 32 sub-module regions (figure 6e). After the elimination process, the remaining candidate areas enter a tracker which monitors their behavior over time.

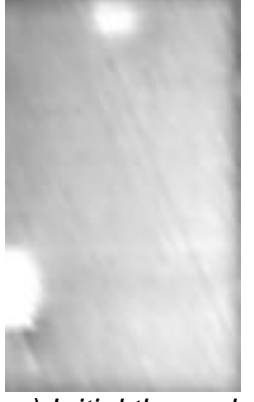

a) Initial thermal map

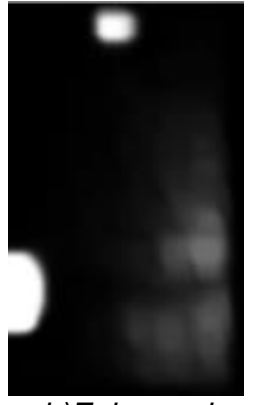

b)Enhanced image

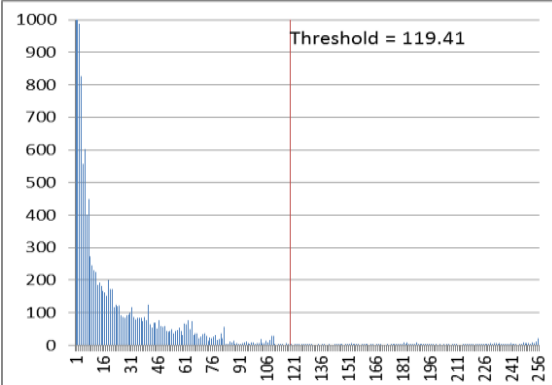

c)Histogram and calculated threshold value

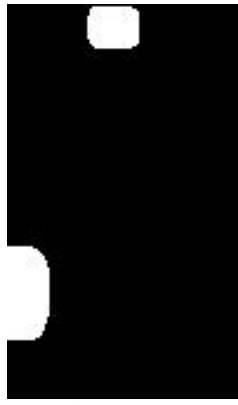

d) Segmented image

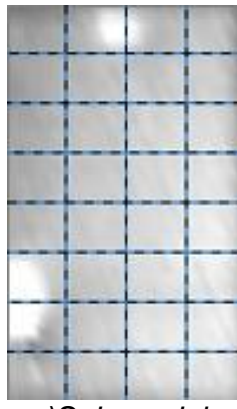

e)Sub-module definition

Fig. 6. Defect segmentation procedure

The described procedure is repeated in each frame and for all the detected PV modules. For a new set of possible defected areas, the matching process follows a minimum Euclidean distance rule to declare the best matches to the currently tracked areas. Thereafter, kalman filters [32] are being used to estimate and update tracked areas position preventing noisy measurements to drastically alter their characteristics. In case that an area is being continuously tracked for more than 30 seconds, it is considered as a valid tracked observation. For each PV module, an aggregation matrix is created every 30 minutes. Aggregation matrices are being populated with information derived from valid tracked areas on the assumption that the previously described holistic and individual conditions (table 1) are satisfied:

$$
A_{k(x, y)}=\left\{\begin{array}{ll}
\sum_{i=0}^{n} I_{(x, y)} \times M_{(x, y)} & \text { if } \mathrm{C}_{1}, \mathrm{C}_{2}, \mathrm{C}_{3} \text { are satisfied } \\
0 & \text { otherwise }
\end{array}\right\}
$$

where $A_{(x, y)}, I_{(x, y)}, M_{(x, y)}$ refer to a pixel located at $(\mathrm{x}, \mathrm{y})$ inside the aggregation matrix, the initial thermal map and the binary mask of valid tracked areas respectively, $n$ defines the 30 minute deadline for the current, $k$, aggregation process. In the particular case that the average PV module intensity is not within the acceptable limits, our method also excludes the neighboring PV modules from being evaluated on the basis that there is a high possibility to be affected by the same underlying cause (e.g. passing cloud). This brings neighborhood-based aspects into the proposed algorithm. Supposing that a tracked area fails to be distinguished for more than 30 seconds, it is being dropped by the tracker. Every aggregation matrix is associated with a condition satisfaction rate (CSR) that is defined as follows:

$$
C S R_{k}=\frac{M}{N}
$$

where $M$ is the number of evaluated frames based on Eq. (9) and $N$ is the number of total incoming frames within the 30 minute period. CSR is used as a confidence factor for the corresponding matrix $k$. By the end of the measuring period that is regulated by the sunset, all PV module's aggregation matrices form a Panel Energy Image (PEI).

$$
P E I_{(x, y)}=\sum_{k=0}^{m} C S R_{k} \times A_{k(x, y)}
$$

where $\mathrm{PEl}_{(x, y)}$ and $\boldsymbol{A}_{(x, y)}$ refer to a pixel located at $(\mathrm{x}, \mathrm{y})$ inside the PEI and the current aggregation matrix $k$ and $m$ defines the number of the aggregation matrices that have been created.

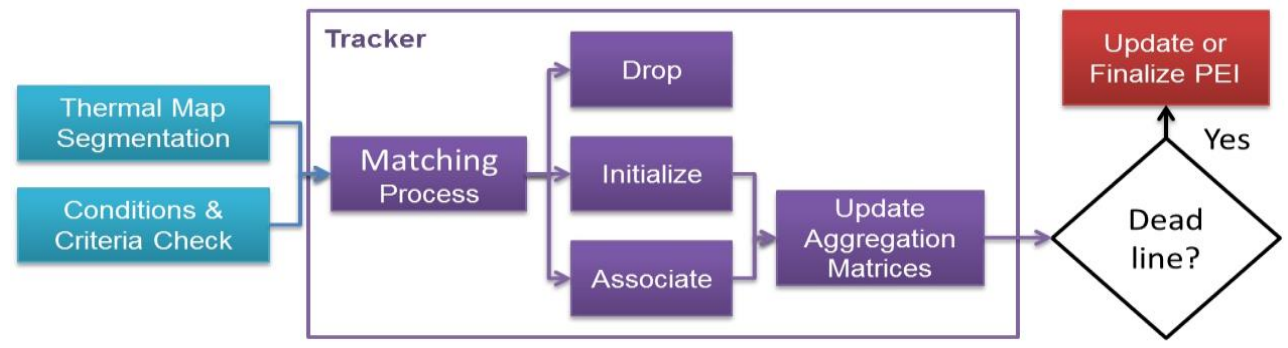

Fig. 7. Panel Energy Image (PEI) Method Overview 


\section{Experimental Set-Up}

The experimental measurements took place in Thermi, Greece, on ten daily sessions spread out between October 2013 and May 2014. The measurements were conducted under varying environmental conditions (Table 2). In order to avoid unwanted specular reflections due to sun or cloud covering that may deteriorate the quality of the captured images the camera was mounted high on a light pole (figure 8a) ensuring that the viewing angle between the inspected $\mathrm{PV}$ modules and the camera is less than $60^{\circ}$. As a result, the protective glass that covers the PV cells has an acceptable emissivity in the $8-14 \mu \mathrm{m}$ waveband. The distance between the PV modules and the camera is approximately $25 \mathrm{~m}$.

Table 2. Ambient environmental conditions during measurements

\begin{tabular}{|l|c|c|c|c|}
\hline & Date & Mean Temperature $\left({ }^{\circ} \mathbf{C}\right)$ & Mean Humidity (\%) & Mean Atmospheric Pressure (hPa) \\
\hline Day1 & $\mathbf{2 3 - 1 0 - 2 0 1 3}$ & 19.94 & 56.47 & 1023.58 \\
\hline Day2 & $\mathbf{2 4 - 1 0 - 2 0 1 3}$ & 20.23 & 57.94 & 1024.82 \\
\hline Day3 & $\mathbf{2 5 - 1 0 - 2 0 1 3}$ & 19.05 & 69.00 & 1022.58 \\
\hline Day4 & $\mathbf{2 9 - 1 0 - 2 0 1 3}$ & 17.33 & 73.77 & 1017.11 \\
\hline Day5 & $\mathbf{3 0 - 1 0 - 2 0 1 3}$ & 17.27 & 75.44 & 1018.11 \\
\hline Day6 & $\mathbf{3 1 - 1 0 - 2 0 1 3}$ & 20.05 & 54.64 & 1021.53 \\
\hline Day7 & $\mathbf{2 8 - 1 - 2 0 1 4}$ & 4.43 & 87.75 & 1001.94 \\
\hline Day8 & $\mathbf{3 - 2 - 2 0 1 4}$ & 7.56 & 56.56 & 1023.12 \\
\hline Day9 & $\mathbf{1 0 - 2 - 2 0 1 4}$ & 14.44 & 55.00 & 1013.94 \\
\hline Day10 & $\mathbf{1 3 - 5 - 2 0 1 4}$ & 23.20 & 52.83 & 1013.00 \\
\hline
\end{tabular}

The mounted imager is equipped with an uncooled microbolometer detector of $25 \mu \mathrm{m}$ pitch that has a resolution of $320 \times 240$ pixels and a field of view of $34^{\circ}$ (horizontal) $\times 28^{\circ}$ (vertical). In addition, its sensitivity is less than $50 \mathrm{mK}$ within the spectral range of 7.5 to $13.5 \mu \mathrm{m}$ and can capture up to 25 frames per second. Each of the inspected PV modules consists of 60 polycrystalline silicon $(\mathrm{p}-\mathrm{Si})$ cells with a nominal peak power $\left(\mathrm{P}_{\mathrm{mpp}}\right)$ of $225 \mathrm{~W}$, peak power voltage of $\left(\mathrm{V}_{\mathrm{mpp}}\right)$ of $29.27 \mathrm{~V}$ and peak power current $\left(\mathrm{I}_{\mathrm{mpp}}\right)$ of $7.81 \mathrm{~A}$. Their dimensions are $166 \mathrm{~cm}$ (height) $\times 98 \mathrm{~cm}$ (width). The modules form a two-string array (figure 8b), with 16 of them laid inside the field of view of the camera. To simulate malfunctioning areas, the PV modules were shaded with leaves, tree branches, dust and dirt resulting in a local temperature increase near the reversed-biased cells (figure $8 \mathrm{c}$ ).

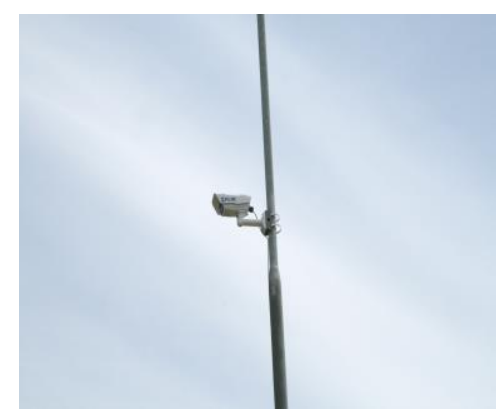

a) Mounted IR camera

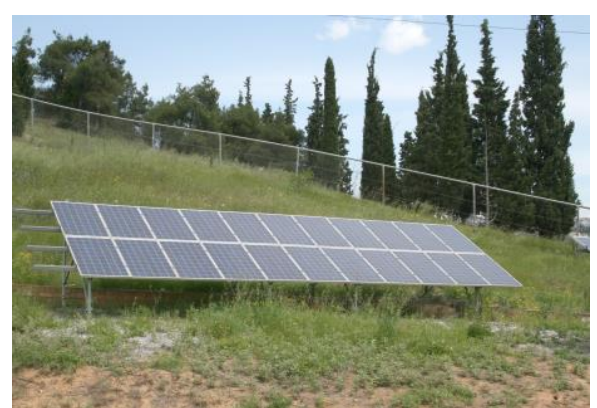

b) The inspected PV modules

Fig. 8. Experimental set-up

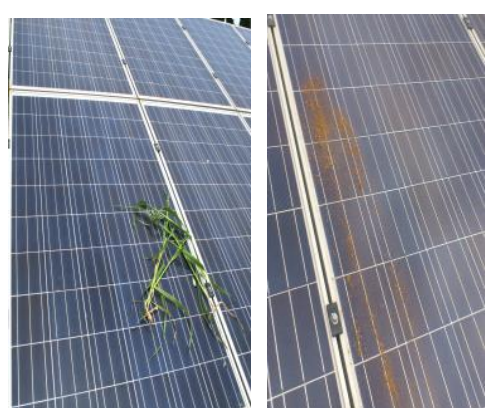

c) Shaded PV modules

\section{Results}

Within this section, the effectiveness of our method is being evaluated as we present its experimental results along with comparisons with existing techniques that emphasize on defect detection in industrial equipment. As already mentioned, the experimental procedure took place on ten daily sessions lasting from sunrise to sunset. The dataset is challenging since the measurements were conducted across different seasons under diversified weather conditions. In order to perform the comparisons, we have manually created ground-truth segmentation images that correspond to the inspected PV modules and their temperature distribution.

Defect detection in PV modules can be considered as a binary classification problem since some areas operate under regular conditions while others are overheated. Junction boxes that are located at the back of the PV modules tend to overheat their adjacent area. Since many defects are associated with junction box overheating, our method attempts to monitor its evolvement and doesn't exempt it. Moreover, on the basis that the PV modules' thermal distribution is constantly fluctuating and is affected by many factors (PV cell operating temperature, ambient temperature, solar irradiation, wind speed, humidity, etc.) within the daytime we adopted a region-based classification approach rather than a pixel-based. As such the sub-module's that were primarily defined for the distinction of accurately detected malfunctioning areas (figure 6e) serve a dual purpose since they contribute to the evaluation procedure as well.

Our method is compared against two baseline approaches that are based on algorithms developed in [27] and [28]. The first baseline method segments each thermal map according to ITADA, whereas the second method uses MFT and $2^{\text {nd }}$ level IBO for the separation of sound and defected areas. Then, the intensity values within the segmented areas populate aggregation matrices that have been created for each baseline method. At the end of the measuring period, 
every PV module holds three matrices that correspond to the results of our method (PEI) and the first and second baseline method (figure 9). It should be noted, however, that the methods developed in [27] and [28] were initially designed for single-image segmentation processes and don't take into consideration any temporal information. The results are shown in figure 10.
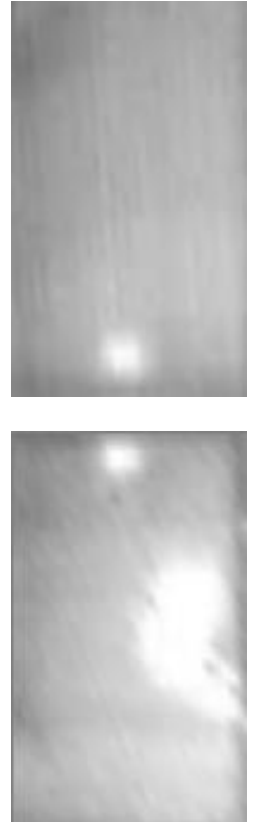

a) Initial thermal map
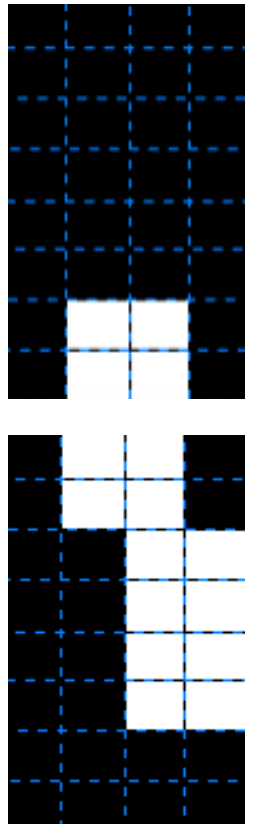

b) Ground-truth
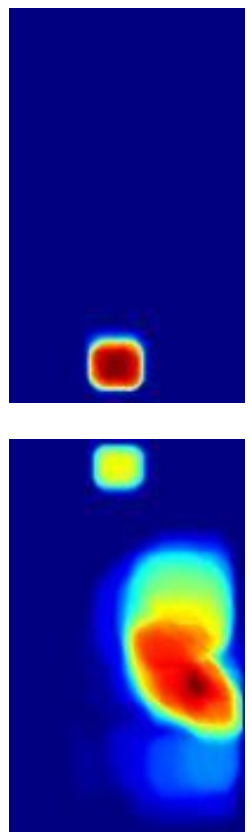

c) PEI
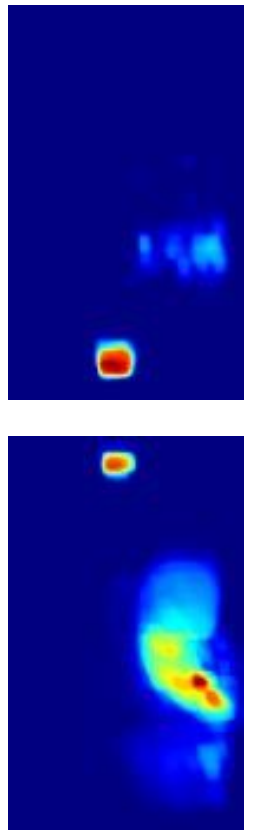

d) Baseline method based on MFT and $2^{\text {nd }}$ level IBO [28]
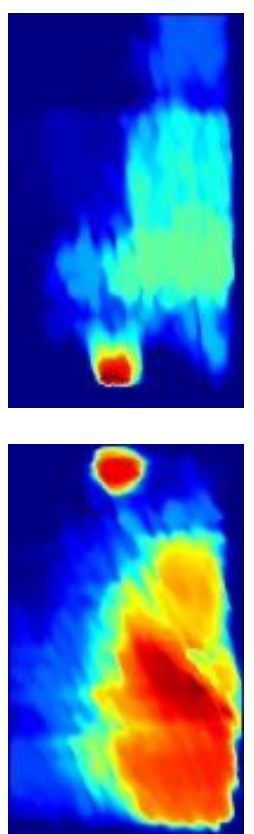

e) Baseline method based on ITADA [27]

Fig. 9. Comparative results of operating PV modules. The second row illustrates a malfunctioning module

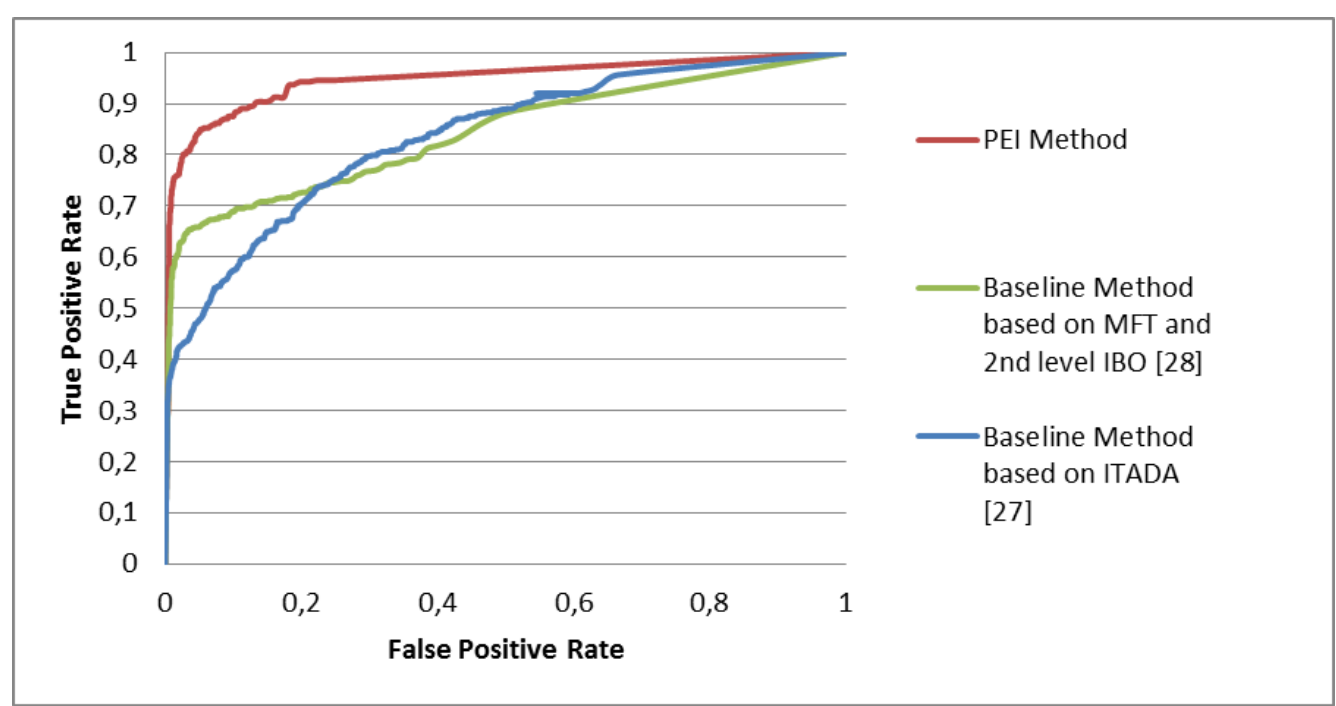

Fig. 10. Classification ROC curve results

Visually, the results of the PEI method seem to be closer to the ground-truth images and exhibit a better resistance to noise that is often caused by specular reflections. The effectiveness of our method is graphically depicted by its ROC curve and its comparison with the baseline methods. What can be also extracted is that in cases where a PV module is seriously defected or an area is notably overheated, the recognition of the area that corresponds to junction box position is getting more challenging, as the algorithm tends to place the threshold in higher intensity values.

From a thermodynamic point of view, ambient temperature plays an important role in the heat transferring process. Additionally, it is well known that it also affects the PV cell operating temperature and alongside its capacity in energy production. Thereby, it is essential to examine ambient temperature's influence on our condition monitoring technique and extract some preliminary conclusions about its impact on defect localization. To this end, we divided our data set into two groups. The first group consists of daily measurements that were conducted in relatively high ambient 
temperature $\left(>19^{\circ} \mathrm{C}\right)$, while the second group includes relatively "colder" daily measurements $\left(<19^{\circ} \mathrm{C}\right)$. As such, days $1,2,3,6$ and 10 belong to the first group and days $4,5,7,8$ and 9 to the second group. The results of the comparison are shown in figure 11.

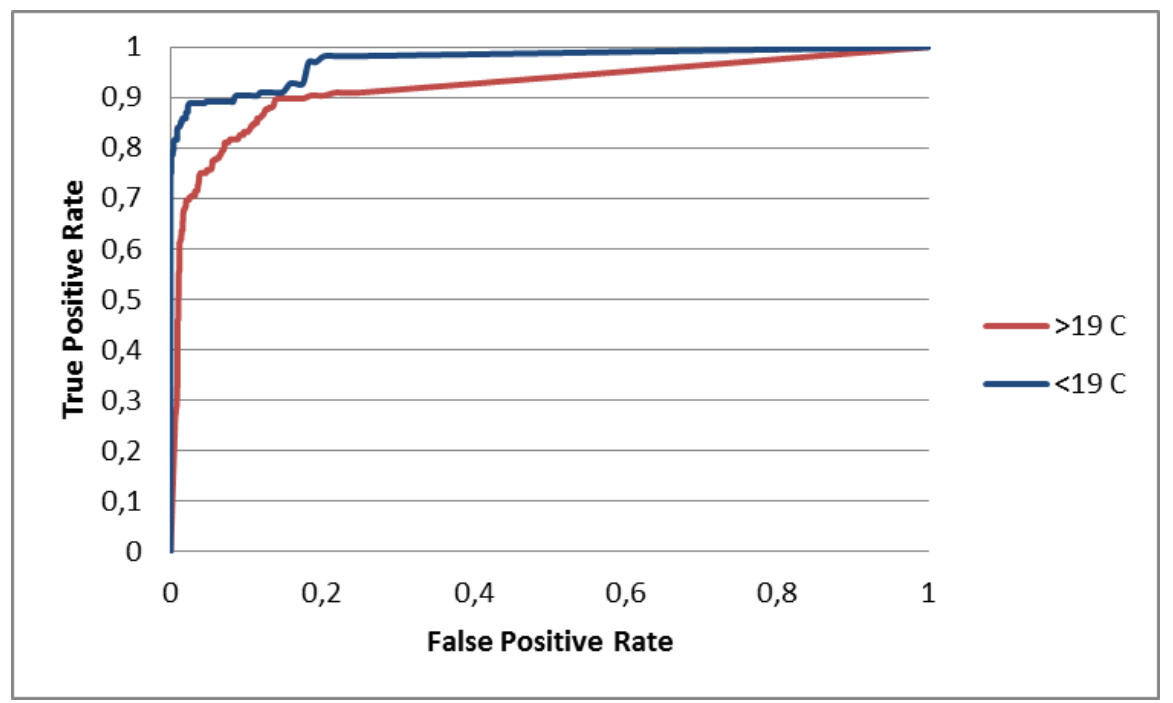

Fig. 11. Roc curve results of PEI method in different ambient temperature conditions

What is clearly distinguishable from the above figure is that temperature is a detrimental factor for the effectiveness of our method. Measurements in relatively low temperatures seem to lead to better results. This is due to the fact that high ambient temperatures tend to create a relatively uniform thermal distribution across the PV modules' surfaces minimizing the gradients between the sound and the defective areas and rendering the defect detection procedure almost impractical.

\section{Conclusions}

In this paper we proposed an early defect diagnosis method, called PEI, that exploits spatio-temporal information from thermal images and monitors the operation of installed PV modules. The first phase of the proposed methodology focuses on the localization of all PV modules that lay inside the IR camera's field of view. This process is performed during nighttime in order to capitalize on the distinctive thermal pattern that characterizes the PV modules. On the other hand, the second phase of our methodology concerns the detection of defected or malfunctioning areas and is designed for daytime operation since the PV modules need to be adequately stimulated. This will allow the visual separation of defected areas which are typically overheated. The segmentation process follows an altered version of the approach that was initially introduced in [28]. A tracking mechanism constantly monitors the progression of the segmented areas over time and gradually aggregates a Panel Energy Image (PEI) with information that derives from the interior of the tracked segmented areas.

After the detailed description of our methodology, experimental results are presented that compare the effectiveness of our approach with baseline methods that emphasize on the detection of defected industrial equipment. The results of our method are very encouraging and outperform the baseline algorithms. Our method performs well and exhibits robustness and efficiency under diversified environmental conditions. Furthermore, it shows adequate resistance to noise and reflections caused by the sun or passing clouds that often affect IR inspections. This reinforces the decision to exploit spatial and temporal information that derives from thermal images and associate it with environmental and neighborhood-based aspects. Some limitations have been identified concerning the detection of junction boxes in cases that another area within the PV module is significantly overheated. Finally, we discussed the effectiveness of our method in different ambient temperature scenarios. The results clearly show that for accurate inspections the ambient temperature needs to be within certain limits since high temperature values tend to obstruct the localization of malfunctioning areas.

Future research efforts should concentrate on more severe PV module defects and their projection in the infrared spectrum over time. Besides, the identification of other key environmental factors that affect IR inspections of PV modules is another significant issue that should be addressed. The association of the identified environmental factors with image quality will determine the optimal ambient conditions for effective vision-based thermal analysis.

\section{Acknowledgments}

This work was partially funded by the national project IPV_Park -Intelligence system for PhotoVoltaic PARK

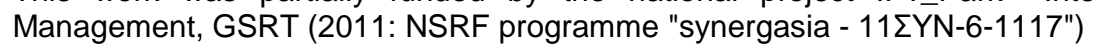




\section{REFERENCES}

[1] Cristaldi, Loredana, et al. "Monitoring of a PV system: The role of the panel model." Applied Measurements for Power Systems (AMPS), 2011 IEEE International Workshop on. IEEE, 2011. [2] Ji,

[2] Young-Hyok, et al. "Maximum power point tracking method for PV array under partially shaded condition." Energy Conversion Congress and Exposition, 2009. ECCE 2009. IEEE. IEEE, 2009.

[3] Buerhop, $\mathrm{Cl}$, et al. "Reliability of IR-imaging of PV-plants under operating conditions." Solar Energy Materials and Solar Cells 107 (2012): 154-164

[4] Maldague, Xavier. "Theory and practice of infrared technology for nondestructive testing." (2001).

[5] Hoppe, Harald, et al. "Quality control of polymer solar modules by lock-in thermography." Journal of Applied Physics 107.1 (2010): 014505.

[6] Breitenstein, Otwin. "Nondestructive local analysis of current-voltage characteristics of solar cells by lock-in thermography." Solar Energy Materials and Solar Cells 95.10 (2011): 2933-2936.

[7] Johnston, Steve, et al. "Temperature-dependent Photoluminescence imaging and characterization of a multicrystalline silicon solar cell defect area."Photovoltaic Specialists Conference (PVSC), 2011 37th IEEE. IEEE, 2011.

[8] Breitenstein, Otwin, et al. "On the detection of shunts in silicon solar cells by photo-and electroluminescence imaging." Progress in Photovoltaics: research and Applications 16.4 (2008): 325-330.

[9] Li, Qingli, et al. "Detection of physical defects in solar cells by hyperspectral imaging technology." Optics \& Laser Technology 42.6 (2010): 1010-1013.

[10] Skoplaki, E., A. G. Boudouvis, and J. A. Palyvos. "A simple correlation for the operating temperature of photovoltaic modules of arbitrary mounting." Solar Energy Materials and Solar Cells 92.11 (2008): 1393-1402.

[11] Botsaris, Pantelis N., and John A. Tsanakas. "Infrared thermography as an estimator technique of a photovoltaic module performance via operating temperature measurements." Proceedings of the 10th ECNDT Conference. 2010.

[12] Acciani, G., G. B. Simione, and S. Vergura. "Thermographic Analysis of Photovoltaic Panels." International Conference on Renewable Energies and Power Quality, Granada/Spain. Vol. 10. 2010.

[13] Jadin, Mohd Shawal, and Soib Taib. "Recent progress in diagnosing the reliability of electrical equipment by using infrared thermography." Infrared Physics \& Technology 55.4 (2012): 236-245.

[14] Sahoo, Prasanna K., S. A. K. C. Soltani, and A. K. C. Wong. "A survey of thresholding techniques." Computer vision, graphics, and image processing41.2 (1988): 233-260.

[15] Otsu, Nobuyuki. "A threshold selection method from gray-level histograms." Automatica 11.285-296 (1975): 23-27.

[16] Minor, Lewis G., and Jack Sklansky. "The detection and segmentation of blobs in infrared images." Systems, Man and Cybernetics, IEEE Transactions on 11.3 (1981): 194-201.

[17] Hamadani, Naser A. "Automatic target cueing in IR imagery." Air Force Interim Report 1 (1981).

[18] Maldague, Xavier, Jean-Claude Krapez, and Denis Poussart. "Thermographic nondestructive evaluation (NDE): an algorithm for automatic defect extraction in infrared images." Systems, Man and Cybernetics, IEEE Transactions on 20.3 (1990): 722-725.

[19] Kamgar-Parsi, Behrooz. "Improved image thresholding for object extraction in IR images." Image Processing, 2001. Proceedings. 2001 International Conference on. Vol. 1. IEEE, 2001.

[20] Chang, Jung-Shiong, et al. "New automatic multi-level thresholding technique for segmentation of thermal images." Image and vision computing 15.1 (1997): 23-34.

[21] Olivo, Jean-Christophe. "Automatic threshold selection using the wavelet transform." CVGIP: Graphical Models and Image Processing 56.3 (1994): 205-218.

[22] Sapina, Rozalija. "Computing textural features based on co-occurrence matrix for infrared images." Image and Signal Processing and Analysis, 2001. ISPA 2001. Proceedings of the 2nd International Symposium on. IEEE, 2001.

[23] $\mathrm{Wu}$, Jin, et al. "Infrared image segmentation via fast fuzzy C-means with spatial information." Robotics and Biomimetics, 2004. ROBIO 2004. IEEE International Conference on. IEEE, 2004.

[24] Silverman, Jerry, Stanley R. Rotman, and Charlene E. Caefer. "Segmentation of multi-dimensional infrared imagery from histograms." Infrared physics \& technology 45.3 (2004): 191-200.

[25] Feng, Du, et al. "Infrared image segmentation with 2-D maximum entropy method based on particle swarm optimization (PSO)." Pattern Recognition Letters 26.5 (2005): 597-603.

[26] Tao, Wenbing, Hai Jin, and Liman Liu. "Object segmentation using ant colony optimization algorithm and fuzzy entropy." Pattern Recognition Letters 28.7 (2007): 788-796.

[27] Chou, Ying-Chieh, and Leehter Yao. "Automatic diagnostic system of electrical equipment using infrared thermography." Soft Computing and Pattern Recognition, 2009. SOCPAR'09. International Conference of. IEEE, 2009.

[28] Heriansyah, Rudi, and S. A. R. Abu-Bakar. "Defect detection in thermal image for nondestructive evaluation of petrochemical equipments." NDT \& E International 42.8 (2009): 729-740.

[29] Giardina, Charles R., and Edward R. Dougherty. "Morphological methods in image and signal processing." Engelwood Cliffs: Prentice Hall, 19881 (1988).

[30] York, Derek. "Least squares fitting of a straight line with correlated errors." Earth and planetary science letters 5 (1968): 320-324.

[31] Nikonorov, A., P. Yakimov, and E. Minaev. "Effective algorithms of flare detection with analysis of the shape in realtime video surveillance systems." Pattern Recognition and Image Analysis 21.3 (2011): 419-422.

[32] Welch, Greg, and Gary Bishop. "An introduction to the Kalman filter." (1995). 